\title{
Effect of Diabetes Mellitus Type II on Long Bones Fractures Healing
}

Ali Sadighi ${ }^{1}$, Mohammadreza Bazavar $^{1}$, Mitra Niafar $^{2}$, Ali Tabrizi $^{3^{*}}$, Nilsa Doorandish $^{4}$

1. Department of Orthopedic Surgery, Shohada Educational Hospital, Tabriz University of Medical Sciences, Tabriz, Iran

2. Department of Endocrinology, Imam Reza Educational Hospital, Tabriz University of Medical Sciences, Tabriz, Iran

3. Students' Research Committee, Tabriz University of Medical Sciences, Tabriz, Iran

4. Department of Internal Medicine, Imam Reza Hospital, Tabriz University of Medical Sciences, Tabriz, Iran

\begin{abstract}
Introduction: Fracture healing is a complex process where synthesis and activation of a cascade of cells and molecules collaborate and participate in regeneration of the fractured bones. There are several factors involved in nonunion of fractured bones. Endocrine and metabolic diseases are regarded as factors causing nonunion of fractured bones. The present study aims at evaluating effect of diabetes type II, as an important and prevalent metabolic disease, on results of surgical treatment of long bones fractures. Method: This case-control study was conducted on 74 patients with isolated fracture of tibia and femur shafts resulting from low-energy trauma. In this study, 50 patients with definite diagnosis of diabetes were compared with 24 metabolically health persons considering age, gender, type of fracture, and treatment method. The diabetic patients were classified in two groups considering their insulin or oral agent. Level of hs-CRP inflammatory marker was also determined in these patients. Union rate and duration as well as relation between inflammatory marker and union rate were studied.

Results: Prevalence of nonunion and delayed union were seen in $8(32 \%)$ and $2(8 \%)$ patients with orally-treated diabetes, respectively. It was $3(12 \%)$ in diabetes patients treated with insulin.

However, there was not any case of nonunion in the health group. There was a statistically significant difference between these groups. According to the regression model, hs-CRP level played a significant role considering nonunion prediction ( $P=0.001$, Odd's Ratio=3.4, $C 195 \%: 1.4-4.8)$. Also, type of diabetes treatment had a significant role in predicting nonunion ( $P=0.04$, Odd's Ratio=0.6, $C 195 \%: 0.3-1.4)$. Duration of being affected by diabetes did not play any important role in nonunion prediction.

Conclusion: Prevalence of nonunion in patients with diabetes suffering from fracture and undergoing orthopedic surgery is higher than healthy people. It seems that increase of inflammatory markers and oral agents therapy plays an important role in causing and predicting of nonunion in these patients.
\end{abstract}

Keywords: Anti-diabetic; Nonunion; Inflammatory Factors

\section{Corresponding author:}

Ali Tabrizi, MD

Department of Orthopedic Surgery, Shohada Educational Hospital, Tabriz University of Medical Sciences, Tabriz, Iran Tel: +989143130829 E-mail: Ali.Tab.ms@gmail.com

Receive date: 2015-03-25| Accept date: 2014-04-20| Publish date: 2015-05-03

DOI: 10.7575/aiac.abcmed.15.03.03.03

\section{A. I}




\section{Introduction}

Diabetes mellitus (DM) type II is one of the most prevalent metabolic diseases and millions of people suffer from it all over the world (1). With general prevalence of $6.4 \%$, DM type II is known with hyperglycemia and hyperinsulinemia (2). There is a strong relation between osteoporosis and increase of fracture risk in patients with DM type II (3). Osteoporosis leads to less bone strength and is accompanied with increase of fracture risk (3). Hip fracture risk is increased in men with DM type II (relative risk of 2.8 in men vs. 2.1 in women) (3).

Bone fracture healing consists of a cascade of cellular events and biochemical factors finally resulting in complete structural and functional regeneration of the injured skeletal system. During the healing process, injury is seen in $5-10 \%$ of fractures leading to nonunion or delayed union of fractures (4). Therefore, stabilization and immobility of fracture is an important factor in healing process (5). Role of mechanical factors is well known in fracture healing process (5-7). Hematoma is formed immediately after fracture, and fills the fractured area. Additionally, inflammatory responses are seen due to stimulation of the injured bone and cellular proliferation begins. Therefore, activation of mesenchymal cells, soft tissue and bone surrounding the fractured area leads to differentiation of chondroblasts and production of fibrocartilage and hyaline cartilage (5-8).

Nonunion is an important problem encountered by orthopedic surgeons. It is detected based on the lack of union symptoms in two radiographic plans and clinical evidences during a specific period (9). There is different healing time considering the involved bones. Nonunion may be attributed to injury velocity, intensity of soft tissue damage, type of fracture (open or closed), crushed bone, and bone loss
(10). There are other factors involved in union process including treatment method, infection, and associated diseases. Metabolic and endocrine diseases including thyroid and parathyroid diseases, osteomalacia, estrogen deficiency, Cushing disease, Paget's disease, and malabsorption syndrome, are regarded as important factors in delaying the bone formation and fracture healing process (11). There are a few studies about the role of DM in increasing the nonunion rate of fractures. However, there is no study dealing with the effect of diabetes treatments on surgical outcome and union of fractures. Therefore, the present study aims at evaluating the effects of DM type II on the outcome of surgical treatment of long bones fractures, and determining the effect of diabetes treatments on union rate.

\section{Methods and materials}

This case-control study was conducted on 74 patients with fracture of tibia and femur shafts referred to orthopedic trauma center of Tabriz University of Medical Sciences from 2010 to 2013. The study was approved by the local medical ethical committee of the Tabriz University of Medical Sciences. Informed consent was obtained from each subject in patient and control groups prior to the study. The traumatic patients had isolated and noncomminuted fractures in their tibia and femur shafts due to low energy trauma resulting from falling. The patients were matched considering the fracture type, surgical treatment, age, and gender. In this study, 50 patients with diabetic criteria were placed in two groups considering the type of DM treatment (oral agent or insulin). In addition, control group consisted of 24 non-diabetic patients with factures but without any other medical diseases. The identified diabetic patients $(n=25)$ had the disease for more than two years and participated in the study based on the type of 
their oral treatment including metformin (1.5$2 \mathrm{~g}$ per day) and glibenclamide (15-20mg). The other group consisted of 25 patients treated with insulin (without any oral agent). The patients were clinically examined, and vascular Doppler was performed (if required) to detect neuropathy and vascular disorders in lower limbs. The patients were excluded if any evidence of neuropathy and macrovascular complications was present. Moreover, patients with open fractures, crushed fractures, soft tissue damage, any systemic diseases as well as patients with history of smoking, consumption of alcohol and mineral supplements during last six months and those older than 50 years were excluded from the study.

The patients underwent orthopedic surgery including open reduction and internal fixation using intramedullary nails with similar method and based on their indications. Before surgery, all patients were put on antibiotic prophylaxis with intravascular injection of cefazolin which continued at least for 24 hours after surgery. The patients were continuously followed up for 9 months (biweekly during the first month and every month thereafter for 9 months) to evaluate union based on radiographic evidences and clinical examinations. The patients of all three groups demonstrated the same rehabilitation during the first week and relative weight tolerance was seen in lower limb fractures after two days. Radiographic evidences confirmed union if it was seen at least in two plans and clinical examinations. Nonunion cases were studied with regard to infection as the first step; white blood cell count, erythrocyte sedimentation rate, and highly-sensitive C-reactive protein (hs-CRP) were checked and the patients were excluded from the study in case of observing any signs of infectious nonunion.

A $20-\mathrm{mL}$ venous blood sample was taken from each patient after an overnight fasting (>8 hours) by a certified phlebotomist using standard laboratory techniques. Blood samples were collected into a serum separator tube (SST; BD Vacutainer ${ }^{\circledR}$, Becton, Dickinson and Company, Franklin Lakes, NJ) for analysis of lipids and glucose. After complete coagulation (30-45 minutes), the SST was centrifuged at 2500 RPM for 30 minutes. The serum was transferred from the spun SST into three labelled plastic tubes: the first tube was used for glucose analysis, the second for lipid panel, and the third tube was stored at $-70^{\circ} \mathrm{C}$ to be used later for hs-CRP measured using an immunoturbidimetric assay system.

Waist circumference was measured midway between the lowest rib and iliac crest with a flexible anthropometric tape. Body mass index (BMI) was calculated as weight ( $\mathrm{kg}$ ) divided by height (m2). Initial examinations of the patients included height measurement $(\mathrm{cm})$ using a wall-mounted height testing instrument with a scale of $1.0 \mathrm{~cm}$, and weight measurement $(\mathrm{kg})$ with a Sega scale. All patients completed a food frequency questionnaire designed and standardized by Iranian Nutrition Institute.

The data were presented as mean \pm standard deviation and $95 \%$ confidence interval. All the statistical analyses were performed using SPSS statistical software version 16.0 (SPSS Inc., Chicago, IL, USA). Normality of the distribution was checked for each variable (one sample Kolmogorov-Smirnov test). Analysis of variance (ANOVA) test was used to check the difference found between means of three groups. Logistic regression model was used to determine the role of factors in fracture union. Chi-square and Fisher's exact tests were used to determine the statistical difference of qualitative variables. A $P$ value $<0.05$ was considered statistically significant.

\section{Results}

The mean age of the patients was $50 \pm 10.4$ years. Seventy four patients including 53 
(71.6\%) male and 21 (28.4\%) female were recruited in the present study. The groups were similar with regard to age, gender, and type of fracture. There were 14 cases of tibia and 10 cases of femur isolated fractures in the case group. Among the diabetic patients, 35 patients had tibia shaft fracture, while 25 cases had femur shaft fracture.

Table 1 shows demographic findings and union rate of three groups. Accordingly, there was a statistically significant difference among three groups considering the nonunion rate $(P=0.001)$. Levels of hs-CRP were $4.3 \pm 2.1$, $2.3 \pm 1.7$, and $0.9 \pm 0.1 \mathrm{mg} / \mathrm{dL}$ in the patients treated with oral agents, insulin, and the control group, respectively ( $\mathrm{P}=0.001)$. According to the regression model, hs-CRP level played a significant role in predicting the nonunion ( $\mathrm{P}=0.001$, $\mathrm{OR}=3.4,95 \% \mathrm{Cl}$ : 1.4-4.8). In addition, the type of diabetes treatment played a significant role considering the prediction of nonunion ( $\mathrm{P}=0.04, \mathrm{OR}=0.6,95 \% \mathrm{Cl}$ : $0.3-1.4)$. Duration of diabetes mellitus did not play a role in predicting the nonunion ( $P>0.05)$. In diabetic patients, there were two cases of dehiscence wound. However, there was no sign of infection in the operated area during the follow-up period.

\section{Discussion}

In lower limbs, nonunion may occur in any bone and it has been reported 3-8\% for tibia and femur fractures. However, open and crushed fractures and fractures with more dislocation demonstrate higher rate of nonunion $(9,12)$. Different factors are involved in the nonunion such as mechanical factors involved in fixation method of fracture. Appropriate stabilization plays an important role in prevention of the nonunion (13).

Metabolic, endocrine, and nutritional factors may affect healing of the fractured bones (13). About $5-10 \%$ of fractures may result in nonunion as a very disabling complication of fractures. In some cases there are clear reasons for nonunion such as surgical factors, operation, and stabilization technique. It is believed that metabolic and endocrine factors are important causes in this regard as well. Principally, nonunion is a multifactorial phenomenon and it is really difficult to find its exact cause. The relation between diabetes mellitus and increase of fracture and osteoporosis risk has been strongly confirmed; diabetes mellitus negatively affects the bone quality and density $(14,15)$. It is stated that hyperglycemia leads to excessive excretion of interleukin 6 (IL-6) from osteoblasts through increasing the insulin activity in bone tissue, thereby enhancing the bone reabsorption by osteoclasts (16). However, there was no relation between $\mathrm{HbA1C}$ percentage and increase in fracture risk. The fracture and surgical treatment results in appearance of inflammatory reaction and, on the other hand, diabetes is associated with increase in inflammatory markers. The more the hs-CRP levels, the more the diabetes complications and risk of cardiovascular diseases. It seems that increase in inflammatory factors and nature of diabetes mellitus affect fracture healing and union processes.

According to the present study, the nonunion rate was higher in diabetic patients rather than the healthy individuals. In a study on different risk factors affecting the nonunion, Hernandez and colleagues found high risk of nonunion in diabetes mellitus type II (OR=2.3, $\mathrm{Cl}$ : 1.4-3.7). Additionally, diabetes type II was associated with increase in fracture healing complications (17). Moreover, Loder suggested that union duration of closed fractures of lower limbs in diabetic patients was 2-3 times more than other people (18). According to Lu et al, insufficient insulin in diabetic patients was associated with less collagen formation in fibroblasts leading to disorder of fracture 


\begin{tabular}{|c|c|c|c|c|}
\hline Variable & $\begin{array}{l}\text { Diabetic patients on } \\
\text { oral agent therapy } \\
\qquad(n=25)\end{array}$ & $\begin{array}{l}\text { Diabetic patients } \\
\text { on insulin therapy } \\
\qquad(n=25)\end{array}$ & $\begin{array}{l}\text { Normal } \\
\text { patients } \\
(n=24)\end{array}$ & $P$ Value \\
\hline Age (year) & \multicolumn{2}{|c|}{$38.8 \pm 8.02$} & $40.1 \pm 7.8$ & 0.2 \\
\hline Gender (M/F) & $7 / 18$ & $6 / 19$ & $8 / 16$ & 0.4 \\
\hline Fasting blood sugar (FBS) & $168.4 \pm 45.4$ & $160.4 \pm 47.3$ & $87.7 \pm 9.4$ & $0.001^{*}$ \\
\hline HbA1C (\%) & $7.8 \pm 1.4$ & $7.2 \pm 1.1$ & - & 0.3 \\
\hline Duration of diabetes & $4.5 \pm 2.1$ & $5 \pm 2.4$ & - & 0.4 \\
\hline Nonunion (\%) & $8(32 \%)$ & $3(12 \%)$ & 0 & $0.001^{*}$ \\
\hline Delay union (\%) & $2(8 \%)$ & 0 & 0 & 0.6 \\
\hline Tibia fractures & $18(72 \%)$ & $17(68 \%)$ & $14(58 \%)$ & 0.1 \\
\hline Femoral fractures & $12(48 \%)$ & $13(52 \%)$ & $10(41.6 \%)$ & 0.1 \\
\hline Time of union (months) & $5.1 \pm 1.7$ & $4.8 \pm 1.5$ & $3.6 \pm 1.2$ & $0.02^{*}$ \\
\hline
\end{tabular}

*Statistically significant

Table 1: Comparison of demographic findings and nonunion rate among three groups

healing process and formation of bone callus in these patients (19). In our study, nonunion in diabetic patients was more frequent than the healthy people.

In the present study, classification of the patients according to their antidiabetic treatment was the most important point. Oral agents such as metformin and glibenclamide were routinely used to treat the patients (20). To better and more control the disease and in conditions of patient's low compliance with the oral agents, insulin is prescribed considering their conditions. It seems that antidiabetic oral agents may affect the fracture healing process and increase the probability of nonunion. In our study, the union duration was longer in the patients treated with antidiabetic oral agents. However, nonunion was seen in only $12 \%$ of patients treated with insulin which was similar to that of healthy people in the previous studies. According to Jeyabalan et al, metformin did not affect bone mass and bone formation process in vitro (21). It seems that metformin does not negatively affect the union process through increasing the sensitivity of tissues to insulin. On the other hand, thiazolidinedions (TZD) were associated with disorders in bone remodeling through increasing the orthopedic complications. According to Lecka-Czernik, TZD resulted in 
disorder in fractures healing process and increased the duration of union. Moreover, there is a strong relation between TZD and bone loss and increase in fracture risk (22). In our study, TZD was not used in any patient. Therefore, high rate of nonunion may not be attributed to the TZD. Higher levels of hs-CRP, higher rate of nonunion and its positive role in predicting the nonunion in patients treated with oral antidiabetic agents might be attributed to active inflammatory process in these patients. Lack of appropriate control and increase in inflammatory markers in the patients treated with oral antidiabetic agents seem to be factors leading to increase in the nonunion rate. Prescription of insulin after the surgical treatment of fractures until the union time may be considered in diabetic patients.
However, further trial studies are required in this regard.

\section{Conclusion}

Prevalence of nonunion in diabetic patients with fracture undergoing orthopedic surgery is higher than the healthy individuals. It seems that increase in inflammatory markers and oral agents therapy play a significant role in causing and predicting the nonunion in diabetic patients.

\section{Acknowledgement}

This study was financially supported by the Students' Research Committee of Tabriz University of Medical Sciences. Department of Endocrinology and Research Deputy of the Faculty of Medicine are appreciated for their cooperation.

\section{References}

1. Roglic G, Unwin N, Bennett PH, Mathers C, Tuomilehto J, Nag S, et al. The burden of mortality attributable to diabetes: realistic estimates for the year 2000. Diabetes Care. 2005;28(9):2130-5.

2. Golozar A, Khademi H, Kamangar F, Poutschi H, Islami F, Abnet CC, et al. Diabetes mellitus and its correlates in an Iranian adult population. PLoS One. 2011;6(10):e26725.

3. Jackuliak P, Payer J. Osteoporosis, fractures, and diabetes. Int J Endocrinol. 2014;2014:820615.

4. den Boer FC, Patka P, Bakker FC, Haarman HM. Current concepts of fracture healing, delayed unions, and nonunions. Osteo Trauma Care. 2002;10(1):1-7.

5. Udupa KN, Prasad GC. Chemical and histochemical studies on the organic constituents in fracture repair in rats. J Bone Joint Surg Br. 1963;45(4):770-9.

6. Bolander ME. Regulation of fracture repair by growth factors. Proc Soc Exp Biol Med. 1992;200(2):165-70.

7. Einhorn TA. Enhancement of fracture-healing. J Bone Joint Surg Am. 1995;77(6):940-56.

8. Einhorn TA, Majeska RJ, Rush EB, Levine PM, Horowitz MC. The expression of cytokine activity by fracture callus. J Bone Miner Res. 1995;10(8):1272-81.

9. Csongradi JJ, Maloney WJ. Ununited lower limb fractures. West J Med. 1989;150(6):675-80.

10. Drosos GI, Bishay M, Karnezis IA, Alegakis AK. Factors affecting fracture healing after intramedullary nailing of the tibial diaphysis for closed and grade I open fractures. J Bone Joint Surg Br. 2006;88(2):227-31.

11. Brinker MR, O'Connor DP, Monla YT, Earthman TP. Metabolic and endocrine abnormalities in patients with nonunions. J Orthop Trauma. 2007;21(8):557-70.

12. Van Demark RE 3rd, Allard B, Van Demark RE Jr. Nonunion of a distal tibial stress fracture associated with vitamin D deficiency: a case report. S D Med. 2010;63(3):87-91, 93.

13. Pourfeizi HH, Tabriz A, Elmi A, Aslani H. Prevalence of vitamin D deficiency and secondary hyperparathyroidism in nonunion of traumatic fractures. Acta Med Iran. 2013;51(10):705-10. 
14. Yamagishi S, Nakamura K, Inoue H. Possible participation of advanced glycation end products in the pathogenesis of osteoporosis in diabetic patients. Med Hypotheses. 2005;65(6):1013-5.

15. Strotmeyer ES, Cauley JA, Schwartz AV, Nevitt MC, Resnick HE, Bauer DC, et al. Nontraumatic fracture risk with diabetes mellitus and impaired fasting glucose in older white and black adults: the health, aging, and body composition study. Arch Intern Med. 2005 25;165(14):1612-7.

16. Wirtz DC, Heller KD, Miltner O, Zilkens KW, Wolff JM. Interleukin-6: a potential inflammatory marker after total joint replacement. Int Orthop. 2000;24(4):194-6.

17. Hernandez RK, Do TP, Critchlow CW, Dent RE, Jick SS. Patient-related risk factors for fracture-healing complications in the United Kingdom General Practice Research Database. Acta Orthop. 2012;83(6):653-60.

18. Loder RT. The influence of diabetes mellitus on the healing of closed fractures. Clin Orthop Relat Res. 1988;(232):210-6.

19. Lu H, Kraut D, Gerstenfeld LC, Graves DT. Diabetes interferes with the bone formation by affecting the expression of transcription factors that regulate osteoblast differentiation. Endocrinology. 2003;144(1):346-52.

20. Aghamohammadzadeh N, Tabrizi A, Niafar M, Najafipor F. Efficacy of adding glutazone to the maximum dose of glibenclamide and metformin resistant on type 2 diabetic patients. Caspian J Intern Med. 2010;1(1):9-11.

21. Jeyabalan J, Viollet B, Smitham P, Ellis SA, Zaman G, Bardin C, et al. The anti-diabetic drug metformin does not affect bone mass in vivo or fracture healing. Osteoporos Int. 2013;24(10):2659-70.

22. Lecka-Czernik B. Bone loss in diabetes: use of antidiabetic thiazolidinediones and secondary osteoporosis. Curr Osteoporos Rep. 2010;8(4):178-84. 